CHRONIC COUGH

\title{
Patients with gastro-oesophageal reflux disease and cough have impaired laryngopharyngeal mechanosensitivity
}

\author{
S Y Phua, L P A McGarvey, M C Ngu, A J Ing
}

Thorax 2005;60:488-491. doi: 10.1136/thx.2004.033894

See end of article for authors' affiliations

Correspondence to Dr L P A McGarvey, Senior Lecturer and Consultant Respiratory Physician, Institute of Clinical Science, The Queen's University of Belfast, Grosvenor Road, Belfast BT1 2 6BJ, UK; I.mcgarvey@qub.ac.uk

Received 1 September 2004 Accepted

10 February 2005
Background: Laryngopharyngeal sensitivity (LPS) is important in preventing pulmonary aspiration and may be impaired by anaesthesia and stroke. It has been suggested that gastro-oesophageal reflux disease (GORD) may also impair LPS, although the underlying mechanism is unclear. The aim of this study was to compare LPS in patients with chronic cough and GORD with healthy subjects and to determine the effect of laryngopharyngeal infusions of both acid and normal saline on LPS.

Methods: Fifteen patients with chronic cough and GORD and 10 healthy subjects without GORD underwent LPS testing using the fibreoptic endoscopic evaluation of swallowing with sensory testing (FEESST) technique. LPS, as measured by the lowest air pressure required to elicit the laryngeal adductor reflex (LAR), was determined both before and after laryngopharyngeal infusions of normal saline and $0.1 \mathrm{~N}$ hydrochloric acid performed on separate days.

Results: The mean baseline LAR threshold of the patient group was significantly higher $(9.5 \mathrm{~mm} \mathrm{Hg}$, range 6.0-10.0) than in normal subjects $(3.68 \mathrm{~mm} \mathrm{Hg}$, range 2.5-5.0; $<<0.01)$. Retest thresholds were not significantly different. In normal subjects LAR thresholds were significantly raised after acid but not after saline infusion $(p=0.005)$. There were no complications associated with the procedure.

Conclusions: Patients with cough and GORD have significantly reduced LPS to air stimuli compared with healthy subjects which could potentially result in an increased risk of aspiration. Exposure to small amounts of acid significantly impaired the sensory integrity of the laryngopharynx.
$\mathrm{L}$ aryngopharyngeal sensitivity (LPS) is important in the prevention of aspiration of materials such as food, liquids, Lor gastric contents into the airway. ${ }^{12}$ LPS may be altered by anaesthesia, head and neck surgery, and neurological impairment. ${ }^{13}$ It has been shown that gastro-oesophageal reflux disease (GORD) may also reduce LPS. ${ }^{4}$ Consequently, this may increase the risk of aspiration of gastric refluxate. Macro or microaspiration has been implicated as one of the mechanisms in the pathogenesis of chronic cough, asthma, and idiopathic interstitial lung disease in patients with GORD. ${ }^{56}$ It is unknown if LPS is altered in patients with GORD and respiratory symptoms, in particular chronic cough.

In a recent study it was found that patients with dysphagia associated with supra-oesophageal reflux had reduced LPS. ${ }^{4}$ A study by Ulualp et al ${ }^{7}$ using pharyngeal water stimulation found that the volume of water required to evoke the pharyngo-upper oesophageal sphincter reflex in subjects with posterior laryngitis associated with GORD was significantly higher than that required for normal subjects, suggesting diminished pharyngeal sensation. It has been documented that this diminished pharyngeal sensation results in the abolition of the pharyngo-upper oesophageal sphincter contractile reflex. ${ }^{8}$ It was concluded that the afferent limb of the reflex may be altered by the pharyngeal acid reflux. ${ }^{7}$ However, the precise relationship between impaired LPS and GORD is unknown.

Assessment of LPS has evolved from subjective methods9 that were difficult to reproduce, to an objective means of determining LPS using air pulse stimulation. Aviv et $a l^{10}$ described the fibreoptic endoscopic evaluation of swallowing with sensory testing (FEESST) method in which air pulses of varying pressures were delivered to the aryepiglottic folds to elicit an involuntary transient adduction of the vocal folds or laryngeal adductor reflex (LAR). Therefore the LAR, mediated by the superior laryngeal nerve and not requiring any conscious and informed response from the patient, has been used as an objective means of measuring LPS. ${ }^{10}{ }^{11}$ Aging and neurological impairments such as stroke are associated with increased air pressure thresholds required to elicit LAR. ${ }^{11}{ }^{12}$ However, the effect of GORD on the LAR is relatively unknown. We hypothesised that LPS as assessed by testing the LAR may also be impaired by the exposure of laryngeal mucosa to acidic refluxate, contributing to an increased risk of pulmonary aspiration of gastric contents.

We have used the FEESST method to determine the differential effects of saline and $0.1 \mathrm{~N}$ hydrochloric acid $(\mathrm{HCl})$ on LAR. To our knowledge this has not previously been performed or reported. The aims of this study were to determine LPS in patients with cough and proven GORD compared with healthy control subjects, and to determine the effect of both acid and normal saline infused into the hypopharynx on LPS in both healthy subjects and those with cough and GORD.

\section{METHODS}

Study subjects

Patients with chronic cough and proven GORD were recruited. GORD was determined on 24 hour ambulatory oesophageal pH studies (Synetics Digitrapper MK II) or upper gastrointestinal endoscopy. All patients had either abnormal GORD parameters on oesophageal $\mathrm{pH}$ study as defined by Johnson and DeMeester ${ }^{13}$ or a minimum endoscopy finding of grade 1 oesophagitis, ${ }^{14}$ and all patients reported improvement although not complete resolution of cough with antireflux therapy. Other common causes for cough, in particular asthma and rhinitis, were excluded on the basis of normal investigations and/or no response to empirical courses of treatment. The control subjects were recruited

Abbreviations: GORD, gastro-oesophageal reflux disease; LAR, laryngeal adductor reflex; LPS, laryngopharyngeal sensitivity 
from volunteers who had responded to advertisements placed in the hospital and university. All the controls had normal 24 hour ambulatory oesophageal $\mathrm{pH}$ monitoring studies. All study subjects were aged between 18-75 years and had no history of previous head and neck surgery, cerebrovascular accidents, or other significant medical illness such as diabetes, ischaemic heart disease, or renal impairment. Patients with proven GORD had stopped taking all proton pump inhibitors and antireflux medications for a minimum of 4 days before beginning the study and remained off medication for its duration.

All subjects gave written informed consent. The study was approved by the Concord Hospital ethics review committee.

\section{LPS testing and determination of LAR threshold}

LPS testing was carried out by endoscopically delivering $50 \mathrm{~ms}$ pulses of air to the arytenoid eminence while varying the pressure intensity of the air pulse in order to elicit the LAR. The air pulses (ranging between 2.0 and $10.0 \mathrm{~mm} \mathrm{Hg}$ in pressure intensity) were delivered by a Pentax AP-4000 air pulse sensory stimulator (Pentax Precision Instruments Corporation, New York, USA). The LAR was identified by direct visualisation of vocal cord adduction on a video monitor receiving a real time image from the fibreoptic endoscope. The protocol is detailed below.

Subjects received local anaesthesia (lidocaine $4 \%$ topical) which was applied to both nares only. No further anaesthesia was used during the study. A nasoendoscope (Pentax FNL-10 AP Fibre-Naso-Pharyngo-Laryngo scope, Pentax Precision Instruments Corporation, New York, USA), $3.2 \mathrm{~mm}$ in diameter, attached to the Pentax AP-4000 air pulse sensory stimulator was then inserted into the nasal passage and the distal tip of the scope was advanced under direct vision into the laryngopharynx until it was located approximately $3 \mathrm{~mm}$ above the arytenoid eminence.

An initial air pulse of $6.0 \mathrm{~mm} \mathrm{Hg}$ was delivered. If LAR was observed, the pressure was reduced in increments of $1.0 \mathrm{~mm} \mathrm{Hg}$ until vocal cord adduction was no longer elicited. The air pressure delivered was then increased by $0.5 \mathrm{~mm} \mathrm{Hg}$ until LAR was observed again. If LAR was not observed at $6.0 \mathrm{~mm} \mathrm{Hg}$, the pressure was increased by $1.0 \mathrm{~mm} \mathrm{Hg}$ until LAR was observed. Once observed, the pressure was reduced by increments of $1.0 \mathrm{~mm} \mathrm{Hg}$ until LAR was no longer elicited. The air pressure was then increased by $0.5 \mathrm{~mm} \mathrm{Hg}$ until LAR was observed again. If no LAR was observed at $10.0 \mathrm{~mm} \mathrm{Hg}$, the procedure was ceased and a threshold of greater than $10.0 \mathrm{~mm} \mathrm{Hg}$ was recorded. The lowest air pressure required to trigger LAR was documented as the threshold LAR pressure. For statistical purposes, the individuals in whom no LAR was observed at the upper limit of $10 \mathrm{~mm} \mathrm{Hg}$ were assigned a LAR threshold of $10 \mathrm{~mm} \mathrm{Hg}$.

\section{LPS testing and LAR determination after hydrochloric acid and saline infusions}

All subjects underwent a randomised, double blind, controlled study during which the LAR threshold to air pulse stimulation was determined using the Pentax AP-4000 air pulse sensory stimulator before and after the infusion of either normal saline (NS) or $0.1 \mathrm{~N} \mathrm{HCl}$. As far as possible, all subjects underwent the procedure at about the same time of day, I week apart. Each subject had both $\mathrm{HCl}$ and NS infused into the pyriform sinus, the order allocated randomly. Both the subjects and the investigators were blind to the fluid (NS or $\mathrm{HCl}$ ) infused. A more complete description of the infusion protocol is described below.

Immediately after the subject's baseline LAR threshold was determined (as described above), a 5 FG nasogastric feeding catheter (Indoplas, Maersk Indoplas PL, Sydney, Australia) was introduced transnasally through the other nostril. With the aid of the nasoendoscope, the catheter was guided and placed in the posterior hypopharynx in the region of the pyriform sinus and used to introduce fluids into the hypopharynx. Solutions used in the study were dyed blue for easy visualisation. The mean $\mathrm{pH}$ of the $\mathrm{HCl}$ solution was 1.2 and of saline was 7.2. Test solutions were delivered at a rate of $1 \mathrm{ml} / \mathrm{min}$, regulated by an IMED 960 (IMED Corporation, San Diego, CA, USA) volumetric infusion pump. Test fluids were infused until an airway protective reflex (that is, a complete adduction of the vocal folds, or swallow, or cough) was elicited. The volume of test solution infused was measured using a digital timer. The catheter was then removed and the LAR threshold was immediately reassessed (as described above).

\section{Statistical analysis}

As the data from LPS testing in this study were not normally distributed, non-parametric statistics were applied. The Mann-Whitney test was used to determine differences in baseline LAR thresholds between patients with GORD and healthy controls. The Wilcoxon signed rank test was used to evaluate differences in LAR threshold in each study group before and after infusion with $\mathrm{HCl}$ or NS. The repeatability of LPS testing was obtained from the baseline measurements in 10 subjects and calculated according to the method described by Bland..$^{15} \mathrm{~A}$ p value of less than 0.05 was considered statistically significant.

\section{RESULTS}

\section{Clinical characteristics of study subjects}

Ten normal subjects and 15 patients with chronic cough and proven GORD were recruited. The clinical characteristics of the study subjects are shown in table 1. All normal subjects underwent 24 hour ambulatory oesophageal $\mathrm{pH}$ monitoring and their results were in the normal range. ${ }^{13}$ Cough patients underwent either 24 hour ambulatory oesophageal $\mathrm{pH}$ monitoring $(\mathrm{n}=11)$ or gastroscopy $(\mathrm{n}=4)$ and met the criteria for GORD. The four patients who underwent gastroscopy were found to have grades 1 and 2 oesophagitis. ${ }^{14}$ On inspection of the larynx during LPS testing it was noted that diffuse laryngeal oedema and posterior commissure hypertrophy was present in nine and 11 of the 15 patients (60\% and $73 \%$, respectively) with GORD. The appearances were unremarkable in the normal subjects.

Table 1 Clinical characteristics of patients with GORD and cough and normal subjects

\begin{tabular}{|c|c|c|}
\hline & $\begin{array}{l}\text { Patients with GORD } \\
\text { and cough } \\
(n=15)\end{array}$ & $\begin{array}{l}\text { Healthy control } \\
\text { subjects } \\
(n=10)\end{array}$ \\
\hline Male & 8 & 3 \\
\hline Mean age (years) & $59 *$ & 34.5 \\
\hline $\begin{array}{l}\text { Age range (years) } \\
24 \text { hour oesophageal } \\
\text { pH monitoring } \\
\% \text { of total time } \mathrm{pH}<4\end{array}$ & 29-72 & $21-65$ \\
\hline Mean (SD) & $7.8(2.2)^{*}$ & $0.74(0.17)$ \\
\hline Range & $2.4-42.9^{*}$ & $0.3-3.9$ \\
\hline $\begin{array}{l}\text { No of reflux events over } \\
24 \text { hours }\end{array}$ & & \\
\hline Mean & $56.3^{*}$ & 14.8 \\
\hline Range & $42-112$ & $3-26$ \\
\hline Endoscopy grading ${ }^{14}$ & & \\
\hline Normal (grade 0) & - & - \\
\hline $\begin{array}{l}\text { Mild to moderate } \\
\text { (grade 1-2) }\end{array}$ & 4 & - \\
\hline Severe (grade 3-4) & - & - \\
\hline
\end{tabular}




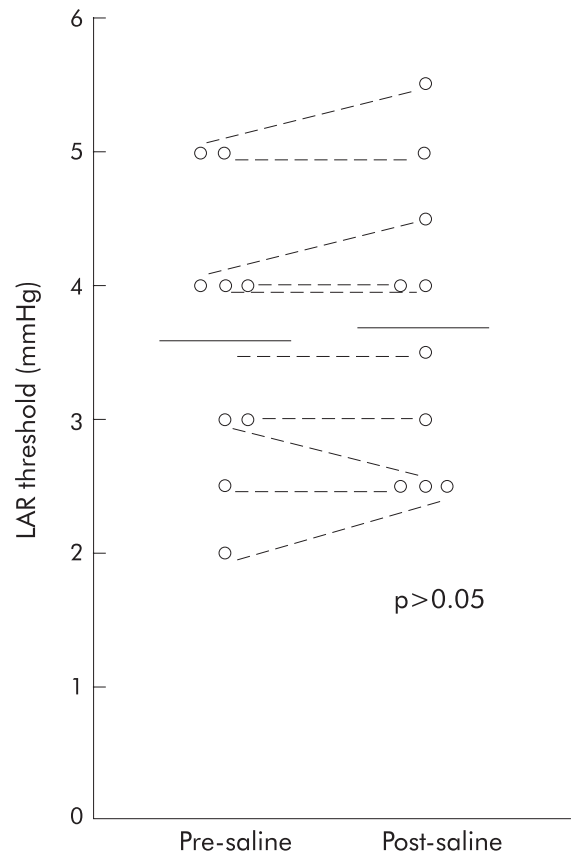

Figure 1 Changes in laryngeal adductor reflex (LAR) in response to infusion of normal saline in healthy control subjects (horizontal bar denotes mean value).

\section{Comparison of LAR thresholds at baseline between study groups}

In patients with GORD the mean LAR threshold was $9.5 \mathrm{~mm} \mathrm{Hg}$ (range 6.0->10). This was significantly higher than the mean LAR threshold of $3.6 \mathrm{~mm} \mathrm{Hg}$ (range 2.5-5.0) determined in control subjects $(\mathrm{p}<0.01)$. In this study the standard deviation for measurement error was 0.34 and the coefficient of variation was $9.7 \%$. Repeatability of the test was 0.96 .

\section{Effect of hydrochloric acid (HCl) or normal saline (NS) on LAR threshold}

Control subjects

LAR thresholds pre and post NS and pre and post $\mathrm{HCl}$ infusion were studied. There was no significant difference in the pre and post NS thresholds $(p>0.05$, fig 1$)$, but a statistically significant difference was found between pre and

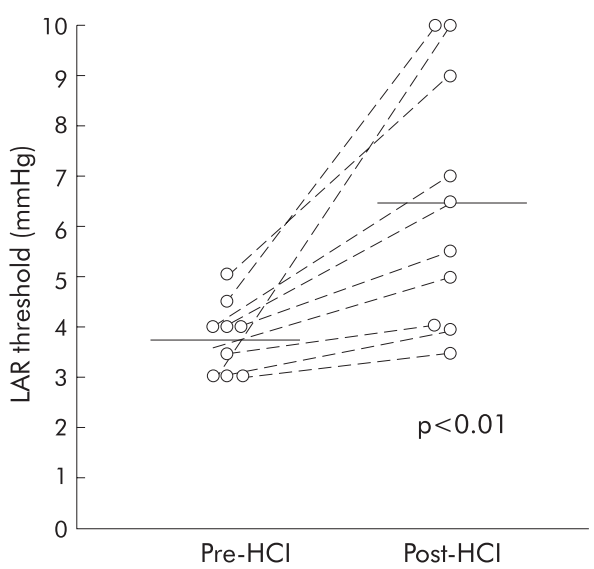

Figure 2 Changes in laryngeal adductor reflex (LAR) in response to infusion of $0.1 \mathrm{~N} \mathrm{HCl}$ in healthy control subjects (horizontal bar denotes mean value). post $\mathrm{HCl}$ thresholds $(\mathrm{p}=0.004$, fig 2$)$. The mean post $\mathrm{HCl}$ threshold was $6.45 \mathrm{~mm} \mathrm{Hg}$. In two subjects a reading of $10.0 \mathrm{~mm} \mathrm{Hg}$ was documented where LAR was still absent at that pressure (the upper limit Pentax AP-4000 air pulse sensory stimulator would deliver).

\section{GORD patients}

Only two subjects with GORD had an LAR threshold $<10 \mathrm{~mm} \mathrm{Hg}$ at baseline. In these subjects LAR thresholds increased from $6.0 \mathrm{~mm} \mathrm{Hg}$ to $6.5 \mathrm{~mm} \mathrm{Hg}$ and from $6.0 \mathrm{~mm} \mathrm{Hg}$ to $7.0 \mathrm{~mm} \mathrm{Hg}$ after saline infusion, and from $6.0 \mathrm{~mm} \mathrm{Hg}$ to $>10 \mathrm{~mm} \mathrm{Hg}$ after $\mathrm{HCl}$ infusion. It was not possible to perform statistical comparisons in thresholds pre and post NS or $\mathrm{HCl}$ in this group.

No subject experienced any adverse reactions to the introduction of $0.1 \mathrm{~N} \mathrm{HCl}$. No episodes of laryngospasm were noted. On repeat nasoendoscopy there were no signs of laryngeal irritation.

\section{DISCUSSION}

In this study we have shown that patients with GORD and cough have increased LAR air pulse thresholds compared with normal subjects. In normal subjects the LAR thresholds were found to be significantly increased following brief exposure to acid, in contrast to the effects of exposure to normal saline. These findings suggest that patients with GORD and cough have decreased laryngopharyngeal sensitivity to mechanostimulation compared with healthy subjects.

Patients are considered to have a normal laryngopharyngeal sensitivity if the LAR can be elicited at less than $4 \mathrm{~mm} \mathrm{Hg}$ of air pressure. A moderate laryngopharyngeal sensory deficit is defined if the LAR is elicited between 4.0 and $6.0 \mathrm{~mm} \mathrm{Hg}$. A patient is considered to have a severe sensory deficit if the LAR is elicited at more than $6.0 \mathrm{~mm} \mathrm{Hg}{ }^{11}$ In our study all subjects with GORD had LAR thresholds greater than $6.0 \mathrm{~mm} \mathrm{Hg}$ (mean $9.0 \mathrm{~mm} \mathrm{Hg}$ ) and could therefore be considered to have a severe LPS deficit. All normal subjects had LAR thresholds in the normal range at baseline. Our results are consistent with a recent study which used a similar method of laryngopharyngeal sensory testing and reported significantly raised LAR thresholds in children (mean age 5 years) with GORD. ${ }^{16}$ Compared to those with normal LAR thresholds, children with highest LAR thresholds in this study had a strong correlative history of recurrent pneumonia.

It is unclear why patients with GORD have diminished LPS to air pulse stimulation, but one possible explanation is that repeated or chronic exposure to acid as a consequence of gastro-oesophageal reflux is responsible. The deleterious effect of acid on mechanosensitivity has been reported previously. ${ }^{17}{ }^{18}$ In one of these studies there was a general desensitisation of oesophageal mechanoreceptors following $\mathrm{HCl}$ application to the oesophagus, and this was particularly evident following repeated application. ${ }^{17}$ The exact mechanism for the change in mechanoreceptor activity and reduced LPS following brief exposure to acid remains unknown. It is possible that the brief exposure results in transient changes in the LAR thresholds with possible permanent increases in patients with chronic GORD. The increased LAR thresholds and thus reduced LPS following acid exposure may potentially increase the risk of pulmonary aspiration. Silent aspiration was associated with impaired LPS in patients following stroke. ${ }^{3}{ }^{4}$ The association between the presence of proximal GORD and disordered swallowing has been further documented in a recent retrospective study on patients with GORD. It was found that patients with GORD had significantly more post swallow residue than age matched normal subjects. ${ }^{16}{ }^{19}$ It was suggested that reduced sensitivity 
was one of the factors in the increased residue. ${ }^{19}$ It is therefore probable that reduced sensitivity to mechanical stimuli in our current patient cohort may also result in swallowing disorders as previously reported in patients with chronic cough, ${ }^{20}$ or increased risk of aspiration. Attenuated LPS in patients with GORD and increased risk of aspiration may also support the hypothesis that respiratory diseases such as asthma, idiopathic pulmonary fibrosis, and chronic cough may be aggravated by aspiration of gastric refluxate.

In our study it was shown that brief exposure to small amounts of $\mathrm{HCl}$ resulted in a significant transient increase in the LAR threshold in normal subjects. No significant increase in LAR was seen with saline. The mean post-acid LAR threshold may well have been significantly underestimated by the fact that the Pentax AP-4000 could not assess LAR thresholds above $10.0 \mathrm{~mm} \mathrm{Hg}$. The limitations of this equipment also meant we were unable to determine the magnitude of any change in LAR thresholds pre and post saline or acid in the majority of GORD patients. Nonetheless, in the two GORD subjects where baseline LAR values $<10 \mathrm{~mm} \mathrm{Hg}$ were recorded, much larger increases in LAR were seen following acid infusion than following saline.

The LAR thresholds to air pulse stimulation obtained in our study were similar to those reported by Aviv et al. ${ }^{11211}$ In their studies the LAR thresholds of control subjects were less than $4 \mathrm{~mm} \mathrm{Hg}^{1}{ }^{10}{ }^{21}$ and LPS was determined by directing the air pulses along the aryepiglottic fold. Similar results were also reported when the thyroarytenoid muscles were stimulated. ${ }^{22}$ With our technique we chose the arytenoid eminence as the point of stimulation. The arytenoid eminence is of close anatomical proximity to the cricopharyngeal junction and is perhaps one of the first areas of laryngeal structures to be exposed to supra-oesophageal reflux of gastric contents. This technique of testing the sensitivity of the arytenoid eminence to air pulses targets the rich pool of sensory nerve endings of the superior laryngeal nerve. Although studies in this area have been confined to guinea pigs, a plexus of such sensory nerve endings has been identified at the arytenoid eminence. ${ }^{23} 24$

Delivering air pulses to the arytenoid eminence proved to be of minimal discomfort to the subjects, and also yielded reproducible results. The reproducibility in eliciting LAR thresholds using our technique was excellent, with no significant difference detected between measurements obtained in the same subjects on different days before the infusion study. The technique is also sensitive to changes in LAR thresholds following brief acid exposure to the laryngopharyngeal area. Due to the limitations of the Pentax AP-4000 air pulse sensory stimulator (where air pressures higher than $10.0 \mathrm{~mm} \mathrm{Hg}$ could not be delivered), it was not possible to determine exact LAR thresholds in most patients with cough and GORD or to measure the changes in LAR thresholds during infusion studies in this group. However, it is clear that LAR thresholds were consistently higher in cough patients than in healthy controls.

A potential criticism of this study is the age difference between healthy control subjects and the patient group. Sensory thresholds using this technique are increased in normal subjects over the age of 60 years, although the magnitude of the increase is very small (approximately $0.5 \mathrm{~mm} \mathrm{Hg}) .{ }^{10}$ We are confident that the higher LAR thresholds to air pulse stimulation observed in GORD patients in our study were much greater than could be attributed to age alone. Although our sample size is small, the results reported provide important insights into upper airway sensitivity in patients with GORD.

\section{Conclusion}

Sensory integrity of the laryngopharynx, which is important in maintaining upper airway protection and the prevention of aspiration, has been shown to be significantly impaired following exposure to small amounts of acid. Patients with cough and proven GORD have reduced laryngopharyngeal sensitivity to air stimuli compared with healthy control subjects and this could potentially result in an increased risk of aspiration.

\section{Authors' affiliations}

S Y Phua, A J Ing, Respiratory Investigation Unit, Department of Thoracic Medicine, Concord Hospital, Sydney, Australia

L P A McGarvey, Department of Medicine, Institute of Clinical Science, The Queen's University, Belfast, UK

M C Ngu, Department of Gastroenterology, Concord Hospital, Sydney, Australia

\section{REFERENCES}

1 Aviv JE, Martin JH, Sacco RL, et al. Supraglottic and pharyngeal sensory abnormalities in stroke patients with dysphagia. Ann Otol Rhinol Laryngol 1996;105:92-7.

2 Shaker R, Milbrath M, Ren J, et al. Esophagopharyngeal distribution of refluxed gastric acid in patients with reflux laryngitis. Gastroenterology 1995; 109:1575-82

3 Aviv JE, Sacco RL, Thomson J, et al. Silent laryngopharyngeal sensory deficits after stroke. Ann Otol Rhinol Laryngol 1997; 106:87-93.

4 Aviv JE, Liu H, Parides $M$, et al. Laryngopharyngeal sensory deficits in patients with laryngopharyngeal reflux and dysphagia. Ann Otol Rhinol Laryngol 2000; 109:1000-6.

5 Harding SM, Richter JE. The role of gastroesophageal reflux in chronic cough and asthma. Chest 1997;111:1389-402.

6 Ing AJ. Interstitial lung disease and gastroesophageal reflux. Am J Med 2001;111(Suppl 8A):41-4S

7 Ulualp SO, Toohill RJ, Kern M, et al. Pharyngo-UES contractile reflex in patients with posterior laryngitis. Laryngoscope 1998;108:1354-7.

8 Shaker R, Hogan WJ. Reflex-mediated enhancement of airway protective mechanisms. Am J Med 2000;108(Suppl 4a):8-14S.

9 Kidd D, Lawson J, Nesbitt R, et al. Aspiration in acute stroke: a clinical study with videofluoroscopy. Q J Med 1993;86:825-9.

10 Aviv JE, Martin JH, Keen MS, et al. Air pulse quantification of supraglottic and pharyngeal sensation: a new technique. Ann Otol Rhinol Laryngol 1993; 102:777-80.

11 Aviv JE, Martin JH, Kim T, et al. Laryngopharyngeal sensory discrimination testing and the laryngeal adductor reflex. Ann Otol Rhinol Laryngol 1999; 108:725-30.

12 Aviv JE, Martin JH, Jones ME, et al. Age-related changes in pharyngeal and supraglottic sensation. Ann Otol Rhinol Laryngol 1994; 103:749-52.

13 Johnson LF, DeMeester TR. Development of the 24-hour intraesophageal pH monitoring composite scoring system. J Clin Gastroenterol 1986;8(Suppl 1):52-8.

14 Savary M, Miller G. The oesophagus. Handbook and atlas of endoscopy. Solothrun, Switzerland: Verlag Gassmann, 1978

15 Bland M. Clinical measurement. In: Bland M, eds. An introduction to medical statistics.3rd ed. Oxford: University Press, 2000:269-72.

16 Link DT, Willging JP, Miller CK, et al. Pediatric laryngopharyngeal sensory testing during flexible endoscopic evaluation of swallowing: feasible and correlative. Ann Otol Rhinol Laryngol 2000;109:899-905.

17 Fass R, Naliboff B, Higa L, et al. Differential effect of long-term esophageal acid exposure on mechanosensitivity and chemosensitivity in humans. Gastroenterology 1998;115:1363-73.

18 Page AJ, Blackshaw LA. An in vitro study of the properties of vagal afferent fibres innervating the ferret oesophagus and stomach. J Physiol 1998;512:907-16.

19 Mendell DA, Logemann JA. A retrospective analysis of the pharyngeal swallow in patients with a clinical diagnosis of GERD compared with normal controls: a pilot study. Dysphagia 2002;17:220-6.

20 Kastelik JA, Redington AE, Aziz I, et al. Abnormal oesophageal motility in patients with chronic cough. Thorax 2003;58:699-702.

21 Aviv JE. Sensory discrimination in the larynx and hypopharynx. Otolaryngol Head Neck Surg 1997; 116:331-4.

22 Bhabu P, Poletto C, Mann E, et al. Thyroarytenoid muscle responses to air pressure stimulation of the laryngeal mucosa in humans. Ann Otol Rhinol Laryngol 2003;112:834-40.

23 Yoshida $Y$, Tanaka $Y$, Hirano $M$, et al. Sensory innervation of the pharynx and larynx. Am J Med 2000;108(Suppl 4a):51-61S.

24 Bradley RM. Sensory receptors of the larynx. Am J Med 2000; 108(Suppl 4a): $47-50 S$. 